\title{
Methods of obtaining instantaneous modulus of viscoelastic solids using displacement-controlled instrumented indentation with axisymmetric indenters of arbitrary smooth profiles
}

\author{
Yang-Tse Cheng ${ }^{\mathrm{a}, *}$, Che-Min Cheng ${ }^{\mathrm{b}}$, Wangyang $\mathrm{Ni}^{\mathrm{c}}$ \\ ${ }^{a}$ Materials and Processes Laboratory, General Motors Research and Development Center, Warren, MI 48090, USA \\ ${ }^{\mathrm{b}}$ Institute of Mechanics, Chinese Academy of Sciences, Beijing 100080, China \\ ${ }^{\mathrm{c}}$ Brown University, Providence, RI, USA
}

Received 8 July 2005; received in revised form 24 August 2005; accepted 10 September 2005

\begin{abstract}
We derive a relationship between the initial unloading slope, contact depth, and the instantaneous relaxation modulus for displacement-controlled indentation in linear viscoelastic solids by a rigid indenter with an arbitrary axisymmetric smooth profile. While the same expression is well known for indentation in elastic and in elastic-plastic solids, we show that it is also true for indentation in linear viscoelastic solids, provided that the unloading rate is sufficiently fast. When the unloading rate is slow, a "hold" period between loading and unloading can be used to provide a correction term for the initial unloading slope equation. Finite element calculations are used to illustrate the methods of fast unloading and "hold-at-the-maximum-indenter-displacement" for determining the instantaneous modulus using spherical indenters.
\end{abstract}

(C) 2006 Elsevier B.V. All rights reserved.

Keywords: Indentation; Viscoelastic solids; Instantaneous modulus

\section{Introduction}

Instrumented micro- and nano-indentation techniques are playing an important role in the study of small-scale mechanical behavior of "soft" matters, such as polymers, composites, biomaterials, and food products. Since many of these materials exhibit viscoelastic behavior, modeling of indentation into viscoelastic solids forms the basis for analyzing indentation experiments in these materials. Theoretical studies of contacting linear viscoelastic bodies became active since the mid 1950s by the work of Lee [1], Radok [2], Lee and Radok [3], Hunter [4], Graham [5,6], Yang [7], and Ting [8,9]. A number of authors have, in recent years, extended the early work to the analysis of indentation measurements in viscoelastic solids [10-16].

One of the widely used approaches is to obtain the elastic modulus from the initial unloading slope (Fig. 1), $\mathrm{d} F / \mathrm{d} h$, using

\footnotetext{
* Corresponding author. Tel.: +1 810986 0939; fax: +1 8109863091.

E-mail address: yang.t.cheng@gm.com (Y.-T. Cheng).
}

the well-known relationship [17-21],

$$
\frac{\mathrm{d} F}{\mathrm{~d} h}=\frac{4 G}{1-\nu} a=\frac{2 E}{\sqrt{\pi}\left(1-v^{2}\right)} \sqrt{A},
$$

where $G$ is the shear modulus, $E=2 G(1+v)$ the Young's modulus, $v$ the Poisson's ratio, $a$ the contact radius, and $A=\pi a^{2}$ is the contact area. Eq. (1) can be derived from the theory for elastic contacts between flat surfaces and spheres [22], flat punches [22], and conical punches [23]. Furthermore, Sneddon has derived expressions for load, displacement, and contact depth for elastic contacts between a rigid, axisymmetric punch with an arbitrary smooth profile and an elastic half-space [24]. Using Sneddon's results, Pharr et al. [18] showed that Eq. (1) holds true for rigid indenters of arbitrary smooth profiles indenting elastic solids. Eq. (1) has also been applied to indentation experiments where plastic deformation occurs. Doerner and Nix [17] suggested that if the area in contact remains constant during initial unloading, the elastic behavior might be modeled as that of a blunt punch indenting an elastic solid. Oliver and Pharr [19] pointed out that Eq. (1) can be used even when the contact area between the indenter and the solid changes continuously as the indenter is withdrawn and the indenter does not behave like a 


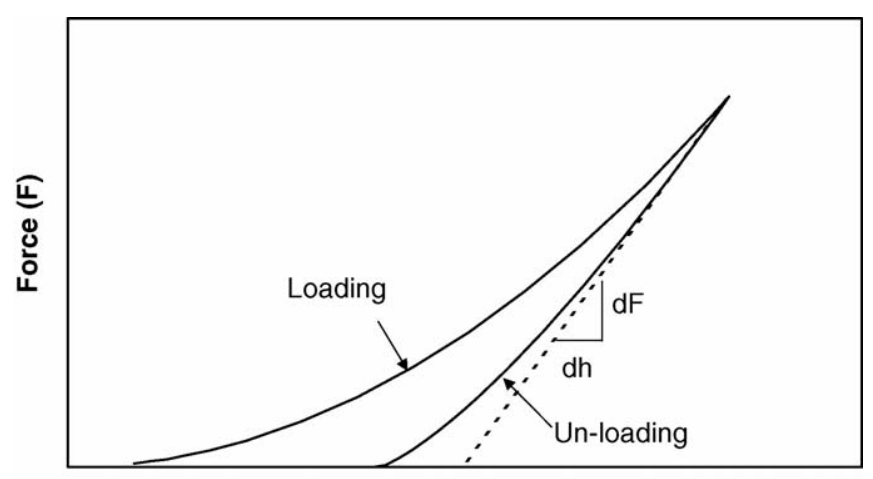

Displacement (h)

Fig. 1. Typical indentation load-displacement curve and initial unloading slope.

flat punch. We have recently shown that Eq. (1) is true for indentation in elastic-plastic solids with or without work hardening and residual stress [25]. On the other hand, Lu et al. [26], and Kumar and Narasimhan [27] have recently suggested that Eq. (1) may not be applicable to indentation in viscoelastic solids.

In order to use Eq. (1), the contact radius, $a$, or contact area, $A$, must be known. For a given indenter, the contact radius or area can be obtained from the contact depth, $h_{\mathrm{c}}$ (see Fig. 2). The most widely used method for estimating the contact depth, $h_{\mathrm{c}}$, is the procedure proposed by Pharr et al. [18] and Oliver and Pharr [19],

$h_{\mathrm{c}}=h_{\mathrm{m}}-\xi \frac{F_{\mathrm{m}}}{(\mathrm{d} F / \mathrm{d} h)_{\mathrm{m}}}$,

where $F_{\mathrm{m}}$ and $(\mathrm{d} F / \mathrm{d} h)_{\mathrm{m}}$ are the respective load and the initial slope of the unloading curve at the indenter displacement, $h_{\mathrm{m}}$. The numerical value of $\xi$ is $(2 / \pi)(\pi-2)=0.727$ and $3 / 4$ for conical and paraboloid of revolution, respectively $[18,19]$. Although Eq. (2) was derived from solutions to elastic contact problems, it has been used to estimate contact depth for indentation in elastic-plastic solids $[18,19]$ and viscoelastic solids

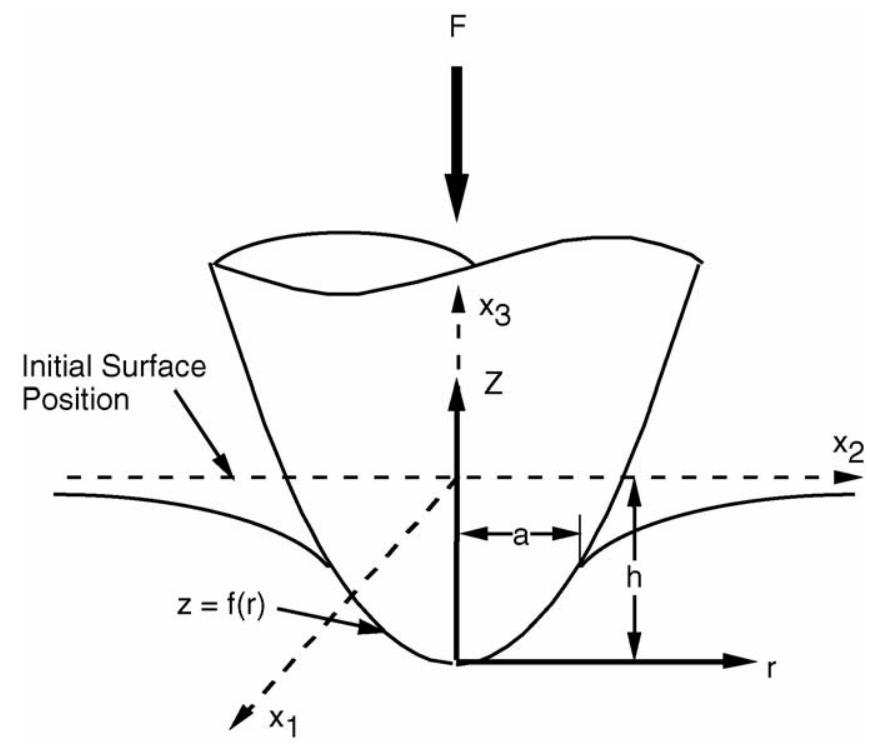

Fig. 2. Illustration of surface deformation by an axisymmetric indenter.
[20,21]. However, our recent work suggests that Eq. (2) may not be applicable for conical and spherical indentation in linear viscoelastic solids under certain loading-unloading protocols [28,29]. In this paper, we examine the applicability of Eqs. (1) and (2) for indentation in viscoelastic solids using axisymmetric indenters of arbitrary smooth profiles.

\section{Analysis}

We consider a rigid, smooth, frictionless, axisymmetric indenter of arbitrary shape, $f(r)$ (Fig. 2) indenting a viscoelastic solid that can be described by the following constitutive relationships [30,31] between deviatoric stress and strain, $S_{i j}$ and $\mathrm{d}_{i j}$, and between dilatational stress and strain, $\sigma_{i i}$ and $\varepsilon_{i i}$,

$$
\begin{aligned}
& s_{i j}(t)=2 \int_{0}^{t} G(t-\tau) \frac{\partial \mathrm{d}_{i j}(\tau)}{\partial \tau} \mathrm{d} \tau, \\
& \sigma_{i i}(t)=3 \int_{0}^{t} K(t-\tau) \frac{\partial \varepsilon_{i i}(\tau)}{\partial \tau} \mathrm{d} \tau,
\end{aligned}
$$

where $G(t)$ is the relaxation modulus in shear and $K(t)$ is the relaxation modulus in dilatation. The time-dependent Young's modulus and Poisson's ratio are then given by $E(t)=9 K(t) G(t) /[3 K(t)+G(t)]$ and $\nu(t)=[E(t) / 2 G(t)]-1$, respectively.

When $G(t), K(t)$, and $\nu(t)$ are time-independent, Eq. (3) reduces to the ones for elastic solids. The corresponding indentation problem has been solved previously, for example, by Sneddon [24], for the contact depth and indenter displacement relationship:

$h=\int_{0}^{1} \frac{f^{\prime}(x)}{\sqrt{1-x^{2}}} \mathrm{~d} x$,

and for the load and displacement relationship:

$F=\frac{4 G a}{1-v} \int_{0}^{1} \frac{x^{2} f^{\prime}(x)}{\sqrt{1-x^{2}}} \mathrm{~d} x$,

where $x=r / a$ and $f^{\prime}(x)=\mathrm{d} f(x) / \mathrm{d} x$. Using these relationships, Pharr et al. [18] derived Eq. (1) for rigid indenters of arbitrary smooth profiles indenting purely elastic solids.

Applying the theories developed by Lee and Radok [3], Graham [5] and Ting [8] to the problem of indentation in viscoelastic solids and assuming time-independent Poisson's ratio, we can write,

$$
\begin{aligned}
& h(t)=\int_{0}^{1} \frac{f^{\prime}(x)}{\sqrt{1-x^{2}}} \mathrm{~d} x, \\
& F(t)=\frac{4}{1-v} \int_{0}^{t} G(t-\tau) \frac{\mathrm{d}}{\mathrm{d} \tau}\left[a(\tau) \int_{0}^{1} \frac{x^{2} f^{\prime}(x)}{\sqrt{1-x^{2}}} \mathrm{~d} x\right] \mathrm{d} \tau .
\end{aligned}
$$

where $x=r / a(t)$.

Eqs. (6) and (7) become the familiar equations for spherical indentation in linear viscoelastic solids. In the "classical spherical" indenter approximation, where the indenter shape is a paraboloid of revolution, where $f(x)=(1 / 2)\left((a x)^{2} / R\right)$ and $R$ 
is the indenter radius, the relationship between contact depth, $h_{\mathrm{c}}(t)$, and the indenter displacement is given by, using Eq. (6),

$h(t)=\frac{a^{2}(t)}{R}=2 h_{\mathrm{c}}(t)$,

and that between force and displacement is given by, using Eq. (7),

$$
\begin{aligned}
F(t) & =\frac{8}{3(1-v) R} \int_{0}^{t} G(t-\tau) \frac{\mathrm{d} a^{3}(\tau)}{\mathrm{d} \tau} \\
& =\frac{8 \sqrt{R}}{3(1-v)} \int_{0}^{t} G(t-\tau) \frac{\mathrm{d} h^{3 / 2}(\tau)}{\mathrm{d} \tau} \mathrm{d} \tau .
\end{aligned}
$$

Eqs. (6)-(9) are special cases of more general expressions derived by Graham [5] and Ting [8]. They showed that Eqs. (8) and (9) are valid when the contact area is a monotonically increasing function of time. The equations for unloading where the contact area decreases monotonically have also been derived $[5,8]$, though they are more complicated. In the following, we use Eqs. (6) and (7) to derive the equation for initial unloading slopes for arbitrary indenter profiles and validate them using finite element calculations.

Differentiating Eq. (7) with respect to time, we obtain

$$
\begin{aligned}
\frac{\mathrm{d} F(t)}{\mathrm{d} t}= & \frac{4}{1-v}\left\{\left.\int_{0}^{t} \frac{\mathrm{d} G}{\mathrm{~d} \eta}\right|_{\eta=t-\tau} \frac{\mathrm{d}}{\mathrm{d} \tau}\left[a(\tau) \int_{0}^{1} \frac{x^{2} f^{\prime}(x)}{\sqrt{1-x^{2}}} \mathrm{~d} x\right] \mathrm{d} \tau\right. \\
& \left.+G(0) \frac{\mathrm{d}}{\mathrm{d} t}\left[a(t) \int_{0}^{1} \frac{x^{2} f^{\prime}(x)}{\sqrt{1-x^{2}}} \mathrm{~d} x\right]\right\}
\end{aligned}
$$

Using Eq. (6), the derivative in the second term of Eq. (10) becomes

$$
\begin{aligned}
& \frac{\mathrm{d}}{\mathrm{d} \tau} a(\tau) \int_{0}^{1} \frac{x^{2} f^{\prime}(x)}{\sqrt{1-x^{2}}} \mathrm{~d} x \\
& \quad=a(\tau) \frac{\mathrm{d} h(\tau)}{\mathrm{d} \tau}+\frac{\mathrm{d} a(\tau)}{\mathrm{d} \tau} h(\tau)-\frac{\mathrm{d} a(\tau)}{\mathrm{d} \tau} \int_{0}^{1} \sqrt{1-x^{2}} f^{\prime}(x) \mathrm{d} x \\
& \quad-a(\tau) \frac{\mathrm{d}}{\mathrm{d} \tau} \int_{0}^{1} \sqrt{1-x^{2}} f^{\prime}(x) \mathrm{d} x
\end{aligned}
$$

We now show that the last three terms on the right-hand side of Eq. (11) cancel each other. Using $x=r / a$ and, and the fundamental theorem of calculus, we obtain

$$
\begin{aligned}
& \frac{\mathrm{d}}{\mathrm{d} \tau} \int_{0}^{1} \sqrt{1-x^{2}} \frac{\mathrm{d} f(x)}{\mathrm{d} x} \mathrm{~d} x \\
& \quad=\int_{r=0}^{r=a(\tau)} \frac{\partial}{\partial \tau}\left(\sqrt{1-\left(\frac{r}{a(\tau)}\right)^{2}}\right) \frac{\mathrm{d} f(r)}{\mathrm{d} r} \mathrm{~d} r .
\end{aligned}
$$

Thus, the last three terms in Eq. (1) indeed cancel each other and Eq. (10) becomes

$$
\begin{aligned}
& \frac{\mathrm{d} F(t)}{\mathrm{d} t} \\
& \quad=\frac{4}{1-v}\left\{\left.\int_{0}^{t} \frac{\mathrm{d} G}{\mathrm{~d} \eta}\right|_{\eta=t-\tau} a(\tau) \frac{\mathrm{d} h(\tau)}{\mathrm{d} \tau} \mathrm{d} \tau+G(0) a(t) \frac{\mathrm{d} h(t)}{\mathrm{d} t}\right\} .
\end{aligned}
$$

Suppose unloading takes place immediately after $t=t_{\mathrm{m}}$ with a constant unloading rate of $\left.(\mathrm{d} h(t) / \mathrm{d} t)\right|_{t_{\mathrm{m}}^{+}}=-v_{\mathrm{h}}$, we obtain using Eq. (14) the initial unloading slope,

$$
\begin{aligned}
\frac{\mathrm{d} F}{\mathrm{~d} h}= & \frac{\mathrm{d} F / \mathrm{d} t}{\mathrm{~d} h / \mathrm{d} t}=\frac{4}{1-v}\left[G(0) a\left(t_{\mathrm{m}}\right)-\left.\frac{1}{v_{\mathrm{h}}} \int_{0}^{t_{\mathrm{m}}} \frac{\mathrm{d} G}{\mathrm{~d} \eta}\right|_{\eta=t_{\mathrm{m}}-\tau} a(\tau)\right. \\
& \left.\times \frac{\mathrm{d} h(\tau)}{\mathrm{d} \tau} \mathrm{d} \tau\right] .
\end{aligned}
$$

When the unloading rate, $v_{\mathrm{h}}$, is sufficiently fast, the second term on the right-hand side approaches zero, independent of the loading history

$$
\frac{\mathrm{d} F}{\mathrm{~d} h}=\frac{4}{1-v} G(0) a\left(t_{\mathrm{m}}\right)=\frac{2 E}{\sqrt{\pi}\left(1-v^{2}\right)} \sqrt{A\left(t_{\mathrm{m}}\right)}
$$

Once this limiting case is reached, Eq. $\left(1^{\prime}\right)$ can be used to determine the "instantaneous" properties, $G(0) /(1-v)$ or $E(0) /\left(1-v^{2}\right)$, provided that the contact depth, $h_{\mathrm{c}}$ or area, $A$, is known as a function of $h_{\mathrm{m}}=h\left(t_{\mathrm{m}}\right)$. The latter condition is provided by Eq. (6) for axisymmetric indenters of arbitrary profiles, which becomes Eq. (8) for spherical indenters. Thus, the unloading slope equation can be used to measure $G(0) /(1-v)$ or $E(0) /\left(1-v^{2}\right)$ by instrumented indentation with axisymmetric indenters of arbitrary smooth profiles, when the unloading rate is sufficiently fast.

When the unloading rate is slow, however, the second term in Eq. (15) is non-negligible and a correction to Eq. (1) is necessary. Since the second term in Eq. (15) is an integral over the entire loading history, it is in general complicated. However, the second term becomes an experimentally measurable quantity for a loading history consisting of a "hold" period between loading and unloading. Specifically, we consider a displacement profile shown in Fig. 3. It has a loading period where the displacement is given by an arbitrary monotonically increasing function of time, a hold period at a constant displacement, and an arbitrary unloading period with an initial unloading rate, $v_{\mathrm{h}}=|\mathrm{d} h / \mathrm{d} t|$. The displacement profile may be described as

$h(t)= \begin{cases}g(t), & 0 \leq t<t_{1} \\ g\left(t_{1}\right), & t_{1} \leq t<t_{\mathrm{m}}\end{cases}$

After evaluating the partial derivative in the integrand and using $x=r / a$, we have

$\frac{\mathrm{d}}{\mathrm{d} \tau} \int_{0}^{1} \sqrt{1-x^{2}} \frac{\mathrm{d} f(x)}{\mathrm{d} x} \mathrm{~d} x=\frac{1}{a(\tau)} \frac{\mathrm{d} a(\tau)}{\mathrm{d} \tau}\left[h(\tau)-\int_{0}^{1} \sqrt{1-x^{2}} \frac{\mathrm{d} f(x)}{\mathrm{d} x} \mathrm{~d} x\right]$. 


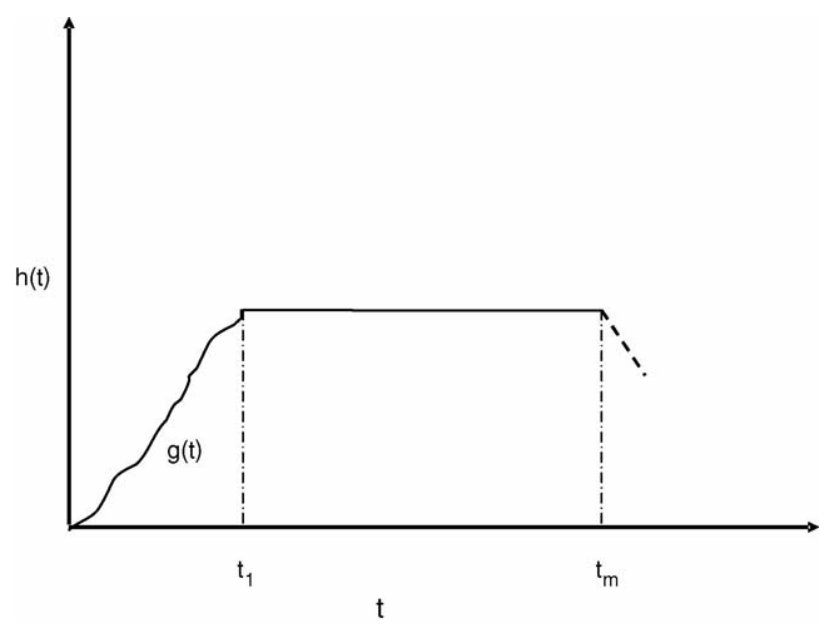

Fig. 3. Illustration of a displacement profile for displacement-controlled indentation.

where $g(t)$ a monotonically increasing function with $g(0)=0$. Inserting Eq. (16) in Eq. (14) and noting that $\mathrm{d} h(\tau) / \mathrm{d} \tau=0$ during the hold period, we obtain the rate of force relaxation at the time of the end of the hold period $\left(t_{\mathrm{m}}^{-}\right)$,

$$
\left.\frac{\mathrm{d} F(t)}{\mathrm{d} t}\right|_{t_{\mathrm{m}}^{-}}=\left.\frac{4}{1-v} \int_{0}^{t_{1}} \frac{\mathrm{d} G}{\mathrm{~d} \eta}\right|_{\eta=t_{\mathrm{m}}^{-}-\tau} a(\tau) \frac{\mathrm{d} g(\tau)}{\mathrm{d} \tau} \mathrm{d} \tau .
$$

Consequently, the initial unloading slope equation, Eq. (15), becomes

$$
\begin{aligned}
& \left.\frac{\mathrm{d} F}{\mathrm{~d} h}\right|_{h=h_{\mathrm{m}}} \\
& \quad=\frac{4}{1-v}\left[G(0) a\left(t_{\mathrm{m}}\right)-\left.\frac{1}{v_{\mathrm{h}}} \int_{0}^{t_{1}} \frac{\mathrm{d} G}{\mathrm{~d} \eta}\right|_{\eta=t_{\mathrm{m}}-\tau} a(\tau) \frac{\mathrm{d} h(\tau)}{\mathrm{d} \tau} \mathrm{d} \tau\right] \\
& =\frac{4}{1-v}\left[G(0) a\left(t_{\mathrm{m}}\right)-\frac{\left.(\mathrm{d} F / \mathrm{d} t)\right|_{t_{\mathrm{m}}^{-}}}{v_{\mathrm{h}}}\right] .
\end{aligned}
$$

Or equivalently,

$$
\frac{4 G(0)}{1-v} a=\frac{2 E(0)}{1-v^{2}} a=\left.\frac{\mathrm{d} F}{\mathrm{~d} h}\right|_{h=h_{\mathrm{m}}}+\frac{\left.(\mathrm{d} F / \mathrm{d} t)\right|_{t_{\mathrm{m}}^{-}}}{v_{\mathrm{h}}}
$$

Eq. (19) shows that $G(0) /(1-v)$ or $E(0) /\left(1-v^{2}\right)$ can be obtained from the measurement of initial unloading slope, $\mathrm{d} F / \mathrm{d} h$, the rate of relaxation of force on the indenter immediately before unloading, $\left.(\mathrm{d} F / \mathrm{d} t)\right|_{t=t_{\mathrm{m}}^{-}}$, and the rate of unloading, $v_{\mathrm{h}}$. The advantage of this "hold at the maximum indentation depth" method is that any rate of unloading, $v_{\mathrm{h}}$, can be used, provided that the force on the indenter is recorded prior to unloading so that $\left.(\mathrm{d} F / \mathrm{d} t)\right|_{t=t_{\mathrm{m}}^{-}}$ is known.

The above analysis is based on the assumption that displacement is the independent variable. When force is the independent variable, a corresponding set of equations have recently been proposed by Ngan et al. using an alternative approach for indentation in linear viscoelastic solids [32-35].

\section{Finite element calculations}

We now demonstrate the validity of Eqs. $\left(1^{\prime}\right)$ and (19) for spherical indentation in linear viscoelastic solids using finite element calculations. We consider a frictionless, rigid spherical indenter of radius $R=2 \mu \mathrm{m}$ indenting an isotropic linear viscoelastic solid. A three-parameter "standard" linear viscoelastic model with a constant Poisson's ratio is used to describe the extension relaxation modulus, $E(t)=2 G(t)(1+v)$,

$E(t)=k_{1}+k_{2} \exp \left(\frac{-t}{\tau}\right)= \begin{cases}k_{1}+k_{2} & \text { for } t \ll \tau, \\ k_{1} & \text { for } t \gg \tau,\end{cases}$

where $\tau$ is the relaxation time. From Eq. (19), we have $E_{0}=k_{1}+k_{2}$ and $E_{\infty}=k_{1}$ for $t=0$ and $t=\infty$, respectively. In this work, we choose a linear viscoelastic material with $E_{0}=696 \mathrm{MPa}, E_{\infty}=68.9 \mathrm{MPa} \tau=0.99 \mathrm{~s}$, and $\nu=0.4833$. The finite element mesh was the same as that used in Ref. [36]. Finite element calculations were carried out using the classical isotropic linear viscoelastic model implemented in ABAQUS [37] using either load or displacement as the independent variable, though only the displacement-controlled indentation results are given in this paper.

\subsection{Method of fast unloading without a hold period}

For constant indentation displacement rate profiles given in Fig. 4a, the corresponding loading-unloading curves from finite element calculations are shown in Fig. 4b. These examples clearly show that, for the same loading history, the initial unloading slopes converge when unloading rate is sufficiently fast, in agreement with Eq. (19). A tangent line with the converged initial unloading slope is also shown in Fig. 4b. Furthermore, finite element results show $G(0) /(1-v)$ or $E(0) /\left(1-v^{2}\right)$ can indeed be obtained using Eq. $\left(1^{\prime}\right)$, thus validating the method of fast unloading $[28,29]$.

\subsection{Method of arbitrary unloading rate with a hold period}

We first study the contact depth for the displacement-control spherical indentation in linear viscoelastic solids with the displacement profile of $10 \mathrm{~s}$ loading, $2 \mathrm{~s}$ holding, $5 \mathrm{~s}$ unloading (see Fig. 5(a)). The maximum indenter displacement is $1 \mu \mathrm{m}$. Fig. 5(b) presents the calculated load-displacement curve for this displacement history. Fig. 5(c) shows the dependence of indentation force $(F)$ and the ratio of contact depth to indentation depth, $h_{\mathrm{c}}(t) / h(t)$, as a function of time. Fig. 5c shows that the force decreases when the indenter position is held constant, as expected from stress relaxation. The calculations also show that $h_{\mathrm{c}}(t) / h(t)$ is a constant $(\approx 0.52)$ during the loading, holding, and at the moment of initial unloading.

This seemingly unintuitive result that, for the displacementcontrolled indentation, the contact depth does not change when the indenter is held at a fixed depth may be understood by considering the hold period as a second loading period with an 

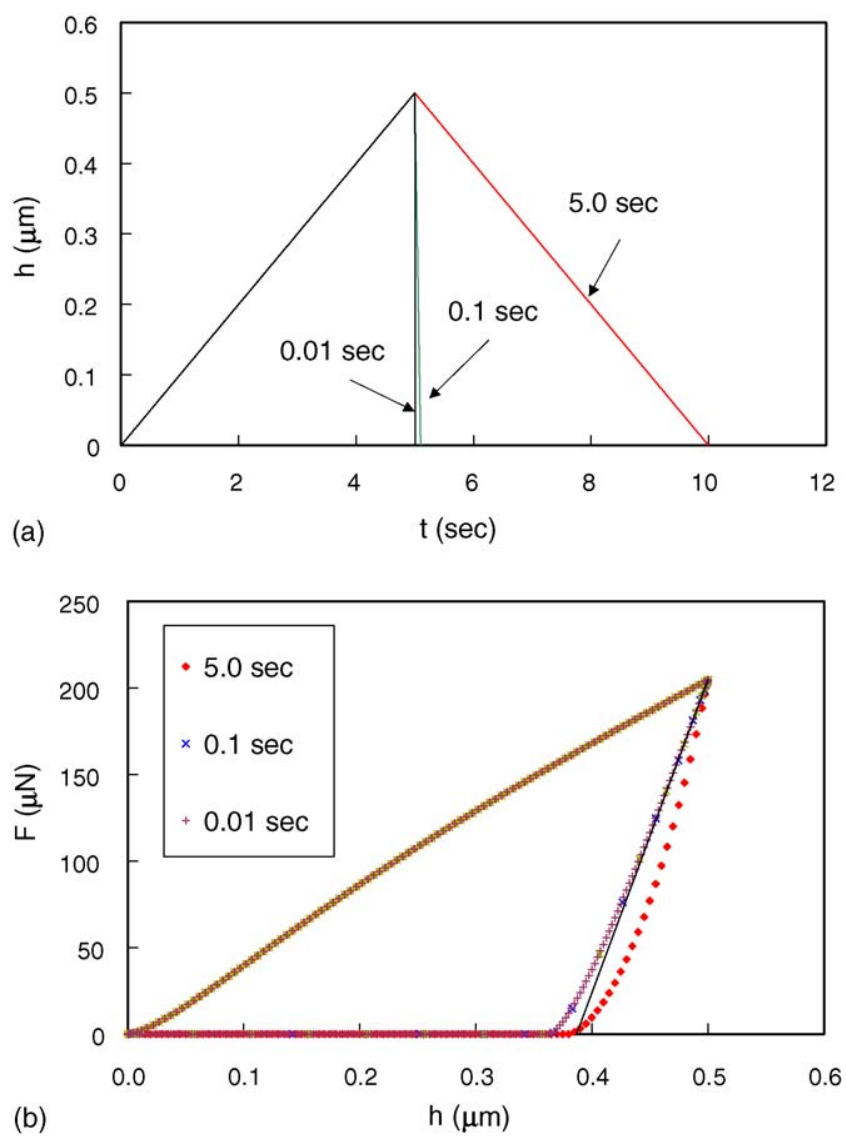

Fig. 4. Displacement-time profiles (a) and the calculated loading-unloading curves (b) for the same loading rate and three different unloading rates. The tangent line with initial unloading slope is also shown for the converged unloading curve (b). The loading-unloading curves are labeled by the time duration of unloading.

infinitesimally small positive indenter displacement rate. During this period, $h_{\mathrm{c}}(t) / h(t)$ is expected to be a constant since the displacement is an increasing function of time. We can also imagine using a very slow loading rate such that the material behaves approximately as a purely elastic solid with a modulus given by $E_{\infty}$. The $h_{\mathrm{c}}(t) / h(t)$ is a constant for both the loading and holding for elastic solids. FEM results also show, as seen from Fig. 5c, that Eq. (8) does not apply to the entire unloading period. In fact, $h_{\mathrm{c}}(t) / h(t)$ decreases with time. Nevertheless, these results confirm that Eq. (8) can be used to obtain the contact depth for the loading and holding periods, as well as at the point of initial unloading for displacement-controlled spherical indentation in linear viscoelastic solids.

We now verify Eq. (19) using finite element calculations. Since $\left.S \equiv(\mathrm{d} F / \mathrm{d} h)\right|_{h=h_{\mathrm{m}}},\left.(\mathrm{d} F / \mathrm{d} t)\right|_{t=t_{\mathrm{m}}^{-}}$, and the contact area, $A$, can be obtained from finite element calculations, the instantaneous modulus $E_{0}^{\text {cal }}$ can then be obtained from Eq. (19) and be compared with the actual instantaneous modulus, $E_{0}$, used as an input parameter for the finite element calculations. Table 1 summarizes the results for displacement-controlled indentation with various loading, holding, and unloading period. Cases I
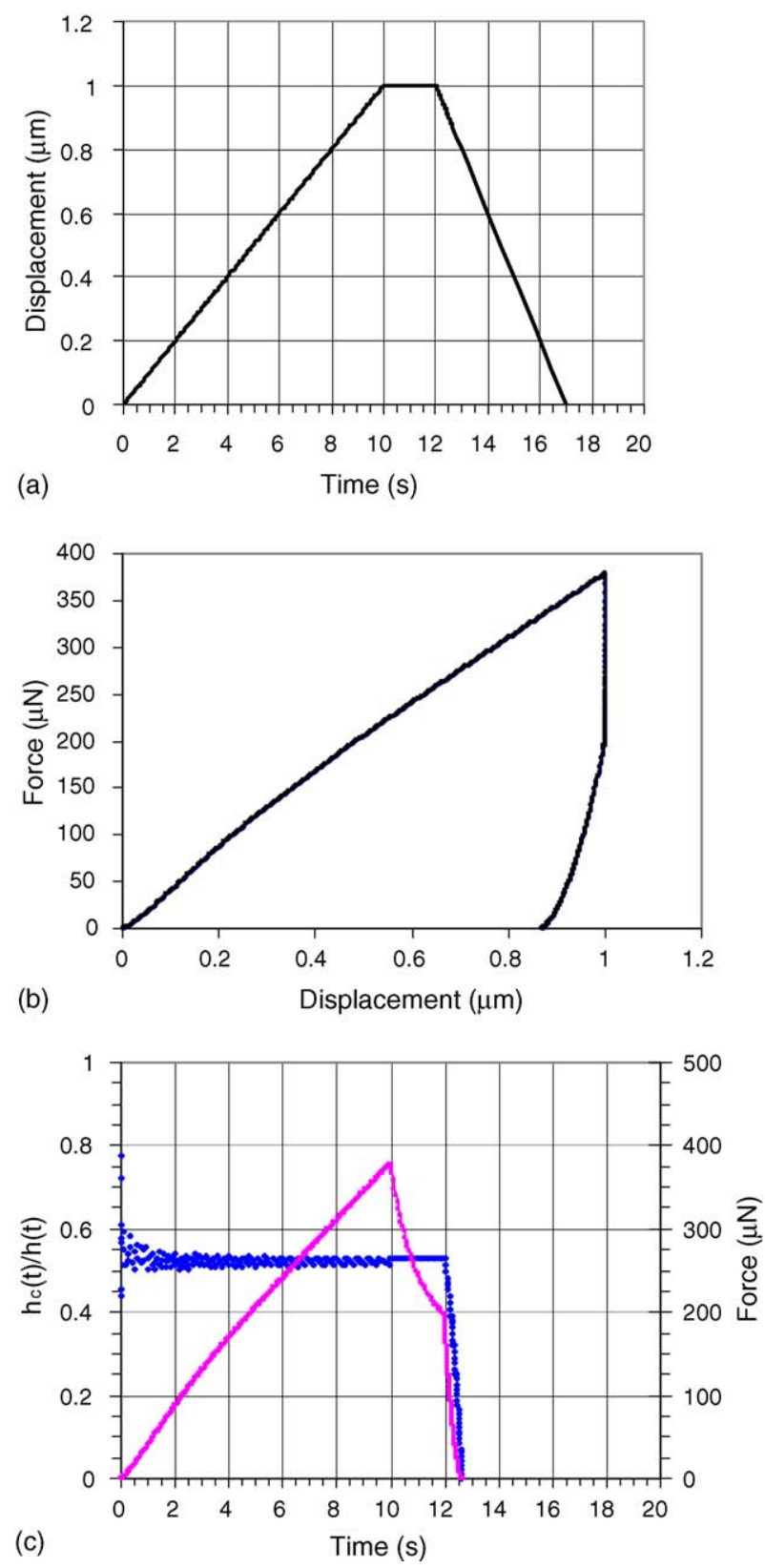

Fig. 5. Displacement profile as the input to the finite element calculation (a), the calculated load-displacement curve (b), and the calculated ratio of contact depth to indenter displacement, $h_{\mathrm{c}}(t) / h(t)$, and the indentation force, $F(t)(\mathrm{c})$.

and II in Table 1 show that when there is no hold segment, the slower the unloading rate, the larger the deviation from the actual instantaneous modulus. However, for the indentation with a hold segment, the instantaneous modulus calculated using Eq. (19) agrees well with the actual value. Thus, Eq. (19) is valid for displacement-controlled spherical indentation in linear viscoelastic solids.

Furthermore, the data in Table 1 show that the contact depth is given by Eq. (8). Contact depths calculated using Eq. (2) can lead to large errors even when the method proposed by Ngan et al. for correcting Eq. (2) is used. 
Table 1

Displacement-controlled indentation in a linear viscoelastic solid using a spherical indenter of $2 \mu \mathrm{m}$ radius

\begin{tabular}{|c|c|c|c|c|c|c|c|c|}
\hline & \multicolumn{8}{|c|}{ Case number } \\
\hline & $\mathrm{I}$ & II & III & IV & $\mathrm{V}$ & VI & VII & VIII \\
\hline Maximum displacement, $h_{\max }(\mu \mathrm{m})$ & 1 & 1 & 1 & 1 & 1 & 1 & 1 & 1 \\
\hline Loading time (s) & 10 & 10 & 10 & 10 & 10 & 10 & 10 & 1 \\
\hline Hold time (s) & 0 & 0 & 5 & 2 & 2 & 10 & 1 & 5 \\
\hline Unloading time (s) & 5 & 0.01 & 5 & 5 & 10 & 1 & 1 & 5 \\
\hline Unloading rate, $v_{\mathrm{h}}(\mu \mathrm{m} / \mathrm{s})$ & 0.2 & 100 & 0.2 & 0.2 & 0.1 & 1 & 1 & 0.2 \\
\hline Apparent stiffness, $S(\mu \mathrm{N} / \mu \mathrm{m})$ & 3501 & 2425 & 2436 & 2571 & 2717 & 2429 & 2524 & 2456 \\
\hline Force relaxation rate, $\left.(\mathrm{d} F / \mathrm{d} t)\right|_{t_{\mathrm{m}}^{-}}(\mu \mathrm{N} / \mathrm{s})$ & - & - & -1.70 & -31.72 & -31.72 & -0.068 & -91.58 & -8.20 \\
\hline Force at unloading, $F_{\max }(\mu \mathrm{N})$ & 378 & 378 & 171 & 198 & 198 & 169 & 246 & 176 \\
\hline Contact area, $A\left(\mu \mathrm{m}^{2}\right)$ & 5.79 & 5.79 & 5.73 & 5.76 & 5.76 & 5.70 & 5.77 & 5.67 \\
\hline Calculated instantaneous modulus, $E_{0}^{\mathrm{cal}}(\mathrm{MPa})$ & 989 & 685 & 689 & 683 & 679 & 691 & 688 & 689 \\
\hline Actual instantaneous modulus, $E_{0}(\mathrm{MPa})$ & 696 & 696 & 696 & 696 & 696 & 696 & 696 & 696 \\
\hline Relative error, $\left(E_{0}^{\mathrm{cal}}-E_{0}\right) / E_{0}(\%)$ & 42 & -1.6 & -1.0 & -1.9 & -2.4 & -0.7 & -1.2 & -1.0 \\
\hline Contact depth by FEM, $h_{\mathrm{c}}(\mu \mathrm{m})$ & 0.531 & 0.531 & 0.525 & 0.528 & 0.528 & 0.521 & 0.529 & 0.519 \\
\hline Average, $h_{\mathrm{c}} / h$ & 0.521 & 0.521 & 0.524 & 0.523 & 0.523 & 0.524 & 0.523 & 0.520 \\
\hline Contact depth by Ngan's method, $h_{\text {Ngan }}(\mu \mathrm{m})$ & 0.919 & 0.883 & 0.947 & 0.939 & 0.938 & 0.948 & 0.924 & 0.945 \\
\hline
\end{tabular}

\section{Summary}

We have derived a relationship between initial unloading slope, contact depth, and instantaneous relaxation modulus for indentation in linear viscoelastic solids by a rigid indenter with an arbitrary axisymmetric smooth profile. This derivation shows that with increasing unloading rate, unloading slope converges to a limiting case given by Eq. $\left(1^{\prime}\right)$. Thus, fast unloading is essential in determining the instantaneous modulus from the initial unloading slope using Eq. $\left(1^{\prime}\right)$. When the unloading rate is slow, a correction to the initial unloading slope equation is necessary. For slow unloading rates, the method of "hold at the maximum indentation depth" can be used to obtain instantaneous modulus using Eq. (19) together with the general equation for contact depth Eq. (6). Finite element calculations have been used to demonstrate both the method of fast unloading and "hold at the maximum indentation depth" for spherical indentation in linear viscoelastic solids.

\section{Acknowledgements}

The authors would like to thank Mike Lukitsch, Yue Qi, Tom Perry, and Wes Capehart, Lou Hector, and Mark W. Verbrugge for valuable discussions. C.-M. Cheng would like to acknowledge partial support from NSF of China, Project No.10372101.

\section{References}

[1] E.H. Lee, Quart. Appl. Math. 13 (1955) 183.

[2] J.R.M. Radok, Quart. Appl. Math. 15 (1957) 198.

[3] E.H. Lee, J.R.M. Radok, J. Appl. Mech. 27 (1960) 438.

[4] S.C. Hunter, J. Mech. Phys. Solids 8 (1960) 219.

[5] G.A.C. Graham, Int. J. Eng. Sci. 3 (1965) 27.

[6] G.A.C. Graham, Int. J. Eng. Sci. 5 (1967) 495.

[7] W.H. Yang, J. Appl. Mech. 33 (1966) 395.

[8] T.C.T. Ting, J. Appl. Mech. 33 (1966) 845.
[9] T.C.T. Ting, J. Appl. Mech. 35 (1968) 248.

[10] L. Cheng, X. Xia, W. Yu, L.E. Scriven, W.W. Gerberich, J. Polym. Sci., Part B: Polym. Phys. 38 (2001) 10.

[11] P.-L. Larrson, S. Carlsson, Polym. Testing 17 (1998) 49.

[12] S. Shimizu, T. Yanagimoto, M. Sakai, J. Mater. Res. 14 (1999) 4075.

[13] M. Sakai, Phil. Mag. A 82 (2002) 1841.

[14] M.L. Oyen, R.F. Cook, J. Mater. Res. 18 (2003) 139

[15] S. Yang, Y.-W. Zhang, K. Zeng, J. Appl. Phys. 95 (2004) 3655.

[16] Y.-T. Cheng, C.-M. Cheng, Mater. Sci. Eng. Rep. A: Rev. J. R44 (2004) 91.

[17] M.F. Doerner, W.D. Nix, J. Mater. Res. 1 (1986) 601.

[18] G.M. Pharr, W.C. Oliver, F.R. Brotzen, J. Mater. Res. 7 (1992) 613.

[19] W.C. Oliver, G.M. Pharr, J. Mater. Res. 7 (1992) 1564.

[20] B.J. Briscoe, L. Fiori, E. Pelillo, J. Phys. D: Appl. Phys. 31 (1998) 2395.

[21] M.R. VanLandingham, J. Res. Natl. Inst. Stand. Technol. 108 (2003) 249.

[22] S.P. Timoshenko, J.N. Goodier, Theory of Elasticity, 3rd ed., McGrawHill, New York, 1970.

[23] A.E. Love, Quart. J. Math. (Oxford Ser.) 10 (1939) 161.

[24] I.N. Sneddon, Int. J. Eng. Sci. 3 (1965) 47.

[25] C.-M. Cheng, Y.-T. Cheng, Appl. Phys. Lett. 71 (1997) 2623.

[26] H. Lu, B. Wang, J. Ma, G. Huang, H. Viswanathan, Mech. TimeDependent Mater. 7 (2003) 189.

[27] M.V.R. Kumar, R. Narasimhan, Curr. Sci. 87 (2004) 1088.

[28] Y.-T. Cheng, C.-M. Cheng, J. Mater. Res. 20 (2005) 1046.

[29] Y.-T. Cheng, C.-M. Cheng, Relationships between initial unloading slope, contact depth, and mechanical properties for spherical indentation in linear viscoelastic solids, GM Research and Development Center Publication R\&D 10,059, February 21, 2005.

[30] W.N. Findley, J.S. Lai, K. Onaran, Creep and Relaxation of Nonlinear Viscoelastic Materials, Dover, Mineola, 1989.

[31] G.T. Mase, G.E. Mase, Continuum Mechanics for Engineers, 2nd ed., CRC, Boca Raton, 1999.

[32] G. Feng, A.H.W. Ngan, J. Mater. Res. 17 (2002) 660.

[33] A.H.W. Ngan, B. Tang, J. Mater. Res. 17 (2002) 2604.

[34] B. Tang, A.H.W. Ngan, J. Mater. Res. 18 (2003) 1141.

[35] A.H.W. Ngan, H.T. Wang, B. Tang, K.Y. Sze, Int. J. Solids Struct. 42 (2005) 1831.

[36] W. Ni, Y.-T. Cheng, C.-M. Cheng, D.-S. Grummon, J. Mater. Res. 19 (2004) 149 .

[37] HKS, Inc., Pawtucket, RI, USA. 